\title{
Exploring interpersonal dynamics between adults and motor disabled children within aesthetic resonant environments
}

\author{
P Lopes-dos-Santos ${ }^{1}$, L M Teixeira ${ }^{2}$, S Silva $^{3}$, M Azeredo $^{4}$ and M Barbosa ${ }^{5}$ \\ ${ }^{1,3,4}$ School of Psychology and Education, University of Porto, \\ Rua Dr. Manuel Pereira da Silva, 4200-392, Porto, PORTUGAL \\ ${ }^{2,5}$ Research Centre for Science and Technology in Art, Portuguese Catholic University, \\ Rua Diogo Botelho, 4169-005, Porto, PORTUGAL \\ 11plsantos@fpce.up.pt, ${ }^{2}$ Iteixeira@porto.ucp.pt, ${ }^{3}$ zanasilva@clix.pt, \\ 4mvza2002@yahoo.com.br, ${ }^{5}$ muflyn@graffiti.net
}

\begin{abstract}
This paper focuses on interpersonal dynamics between the child with disabilities and the adult monitoring his/her performance in Aesthetic Resonance Environments. Drawing upon a social constructivist approach, a framework for human interactivity was checked against empirical data obtained from the exploratory implementation of an environment intending to stimulate body awareness and enhance movement in a group of six children with severe neuromotor disabilities. Results showed that the adult assumed the role of a facilitator, mediating interactions between children and the technological system. The provided social mediation increased quality of movement and improved levels of engagement in the observed group of participants.
\end{abstract}

\section{INTRODUCTION}

The application of the concept of 'aesthetic resonance' within the field of special needs and rehabilitation has been recently documented as a therapeutic tool of potential great value (e.g., Brooks and Hasselblad, 2004). The implementation of Aesthetically Resonant Environments is based on a core of technological resources, which captures and transforms human physical movement in events that produce attractive changes in the proximal multi-sensory environment. Therefore, an Aesthetic Resonance Environment is generated by a responsive technology and refers "(...) to a situation where the response to an intent is so immediate and aesthetically pleasing as to make one forget the physical movement (and often effort) involved in the conveying of the intention” (Brooks, Camurri, et al., 2002, p. 205). Such environments are in line with the key rehabilitation principle, which states that therapy must enable patients to improve their often residual capabilities without causing them unnecessary fatigue and frustration (Kizony, Katz, et al., 2004).

Aesthetically Resonant Environments are assumed to enhance movement through "fun" and aesthetic experience, hence implying a shift from task to play. Regarding more traditional views on rehabilitation, such shift is allowed in a context of non-human interactivity where pleasurable experiences result from the interaction between the individual with disabilities and a (non-human) set of integrated digital devices. The formal role of human interactivity in this framework may become increasingly subsidiary as technological investment is made on individualizing the operating system according to the functionalities, preferences, and needs of each user. Centred on optimizing the relationship between the child with disabilities (C) and the system (S), research on Aesthetically Resonant Environments has paid little attention to the interpersonal dimension.

However, interpersonal processes in Aesthetically Resonant Environments are inherent to the presence of an adult (A) monitoring the child's relation with the system. Even when we conceive the system as a standalone source of feedback, the intervention of the adult is likely to occur during CS (child-system) interactivity. This intervention may simply show itself as the act of introducing the system to the child, but it may also extend to higher levels of involvement (e.g., demonstrating possibilities of gesture, encouraging responsiveness, improving child's performance through assistance and guidance). As Brooks (2004a) contends, “( $\ldots$ ) even notionally single-user activities often happen together with other people and a purely virtual environment tends to cut people off from their surroundings making it difficult for several collocated 
people to share an experience. (...) For these reasons purely virtual environments may not be desirable in cases where the participants have a disability" (p. 89).

As a general aim, our paper attempts to contribute to the expansion of a dyadic CS concept of Aesthetic Resonant Environments into a triadic CSA (child-system-adult) framework. Drawing upon the social constructivist view (e.g., Wertsch, 1985; Rogoff, 1993, 2003), we propose an approach of the adult as a mediator in the relation between the child with disabilities and the responsive technological system. According to this perspective, child performance and interpersonal processes are not specifiable as independent entities. Taking "action in social context" as the unit of analysis requires a relational interpretation where the participation of the adult in shared activities is assumed to provide the necessary structure or scaffolding for the child to elaborate and transform his or her demonstrated skills (Petersson and Brooks, 2006).

Findings reported in the present paper came from a research project in which we designed and implemented an Aesthetically Resonant Environment intending to stimulate body awareness and improve quality of movement in children with severe motor disabilities. Since the interpersonal dimension appeared as an important organizer of children's behaviour during the course of sessions, we decided to look closer at such dimension. Therefore, the more specific goal of this study was to investigate child-adult interactivity variables within the designed Aesthetically Resonant Environment. Did such interactivity affect children's performance? Did social interactive phenomena exert a strong influence on aspects like the quality of gesture or levels of child engagement in the observed sample?

\section{METHOD}

\section{$2.1 \quad$ Participants}

Participants were six children (3 males and 3 females) with severe neuromotor disabilities associated with cognitive and language impairments. Their age ranged from 12 to 13 years old and they were all attending full-time (re)habilitation services in an institution for handicapped children. Inclusion criteria included the capacity to move upper limbs with a reasonable degree of amplitude, the ability to understand simple instructions (verbally or non-verbally) and to perceive contingencies between one's own gestures and the delivered visual/auditory feedback. Parents signed the informed consent.

\subsection{Setup}

Using a colour tracking technique, a responsive digital system was designed that translated local gesture into visual and auditory feedback. Each tracked movement simultaneously generated visual feedback projected onto a screen, and controlled the pitch variation of a MIDI instrument. Several templates were created, with different sorts of stimuli (e.g., notes from different scales versus chords versus percussive sounds). The choice of the program (timbre) was left as an option in the interface, as well as the possibility of shifting or lowering the register of each instrument.

The responsive system was implemented using the Max/MSP programming environment for audio processing components associated with the EyesWeb technology (www.eyesweb.org) for visual components. EyesWeb is an open software platform conceived for multimodal analysis and processing of expressive gesture in movement. Developed at the InfoMus Lab of the University of Genoa, the EyesWeb platform consists of a number of integrated hardware and software modules that can be easily interconnected and expanded. The software modules function as a development environment including several libraries of reusable components, which can be assembled to build patches in a visual programming language.

\subsection{Procedure and Coding System}

Each participant was observed during four sessions that took place on different days at the centre where they were receiving (re)habilitation services. After entering the experimental room, children were comfortably positioned, facing the screen where visual feedback stimuli were projected. Sessions lasted approximately 25 minutes (Mean = 25 min. $30 \mathrm{sec}$; Range = $18 \mathrm{~min}$. $20 \mathrm{sec}$. to $32 \mathrm{~min}$. $10 \mathrm{sec}$.) and were videotaped using simultaneously two cameras placed in different points (one of them -the front camera- captured child's behaviours, and the other -the back camera- taped screen events).

For analysis purposes, each session was divided in successive 10 seconds intervals and behavioural units were scored for their occurrence once (and only once) per interval regardless of the number of onsets during the interval. Some of the units were recorded on an event base (the observer noted the presence or the 
absence of events but ignored their duration) and others were scored on a time base (an event was coded within a given 10-sec interval if it could at least be observed during most of the interval length).

All event base units of the developed coding system apply to the child and are briefly described in Table 1. Full operational definitions, containing detailed behavioral descriptors and examples, are available from the first author.

Table 1 Brief definitions of event base observational categories

\begin{tabular}{ll}
\hline \multicolumn{1}{c}{ Categories } & \multicolumn{1}{c}{ Definition } \\
\hline Non-verbal communication & $\begin{array}{l}\text { Contextually appropriate use of conventionalized forms of } \\
\text { non-verbal signs to express intentions, desires... } \\
\text { Verbal communication }\end{array}$ \\
Positive affect & $\begin{array}{l}\text { Positive facial or vocal expressions (e.g., smiles, laughs) } \\
\text { Negative affect }\end{array}$ \\
Avoidance & $\begin{array}{l}\text { Eehaviour (visual, postural) used to actively avoid contact or } \\
\text { interaction with the adult } \\
\text { Gpontaneous gesture }\end{array}$ \\
Gesture belonging to the child's typical repertoire performed \\
without modelling by the adult \\
Gesture reproducing a gesture performed by the adult \\
Creative movement
\end{tabular}

Concerning time base units, the coding system assessed children's engagement and Child-System-Adult interactivity.

Engagement was broadly defined as the amount of time children spend interacting with the responsive technological system. Taking as reference the level of activity that each child was typically able to demonstrate, four levels of engagement behaviour were considered:

- Nonengaged - The child is passive, unresponsive, and ignoring the adult or the visual/auditory information produced by the technological feedback system.

- Attentional engagement - The child is not actively interacting with the system but is paying attention to the adult while he or she interacts with the system.

- Sparsely active engagement - The child shows intermittent and non-sustained actions as if not fully interested in interacting with the system.

- Active engagement - The child actively interacts with the system performing continuously movements and attending to the delivered feedback.

CSA (Child-System-Adult) interactivity was described by the different interaccional states abstracted from the child and/or the adult behaviour. The following categories were defined:

- Adult as model - The adult is mainly directive while demonstrating the system to the child. Demonstrative movements may just involve actions performed in front of the child, or may include physical contact (e.g., adult manipulates the child's hand to obtain feedback from the system). Verbal instructions are also coded in this category.

- Child-Adult as peers in the system - Both adult and child interact simultaneously with the system by reciprocally imitating or elaborating each other behaviour (e.g., combinations; expansions). Although non-directive, the adult may take some initiatives, providing props, comments on the child's actions or suggestions to extend the child's activity.

- Child-Adult as peers outside the system - Child-adult interactions with no direct intermediation of the technological responsive system (e.g., child engages in play or in direct communication with the adult, "ignoring” the system). 
- Child as performer/Adult as public - The child spontaneously uses the system with the noticeable purpose of being observed and appreciated by the adult. (e.g., before, after or while interacting with the system the child looks at the adult as if waiting for approval or for some other kind of reinforcement).

- No social interaction - When no one of the previous described categories is observed (e.g., the child ignores the adult; the adult is controlling the technological device not paying attention to the child).

In observational research, a principal index of usefulness is the reliability of the used coding system, as measured by interobserver agreement. To establish the reliability of obtained scores, two independent observers coded a total of 120 minutes of the tapes (i.e., $72010 \mathrm{sec}$ intervals). Reliability was determined for event base categories (as a whole), for engagement behaviour and for Child-System-Adult interactivity, and was computed as the total number of agreements divided by the number of agreements plus disagreements. Levels of reliability were $83 \%$ for event base categories, $88 \%$ for engagement behaviour, and $85 \%$ for ChildSystem-Adult interactivity.

\subsection{Data Analysis}

Due to variations in the number of observed intervals, results are described in terms of relative frequencies (RFs). RFs were calculated dividing individual frequencies by the total amount of intervals considered in each unit of analysis. To examine the statistical significance of differences we used non-parametric tests. In the employed tests, original scores are changed from continuous to ordinal scales. As usually recommended in these cases, group results for the dependent variables will not be presented through means but described by their median values.

\section{RESULTS}

\subsection{Child-System-Adult Interactivity}

RFs medians for the coded interactive states in each of the four sessions are presented in Table 2. As results from the Friedman two-way analysis of variance test revealed, lengths of 'No Social Interaction' and of 'Adult as Model' states decreased significantly from session to session.

Table 2. RFs medians for interactive states in the four sessions.

\begin{tabular}{lccccc}
\multicolumn{1}{c}{ Interactive States } & 1rst. & 2nd, & 3rd. & 4th. & Signif. \\
\hline No social interaction & .218 & .139 & .117 & .065 & $\mathrm{p}<.001$ \\
Adult as model & .356 & .313 & .154 & .110 & $\mathrm{p}<.001$ \\
CA as peers in the system & .108 & .173 & .229 & .252 & $\mathrm{p}<.001$ \\
CA as peers outside the system & .077 & .096 & .155 & .220 & $\mathrm{p}<.001$ \\
C as performer/A as public & .253 & .241 & .302 & .348 & $\mathrm{p}<.003$ \\
\hline
\end{tabular}

Inversely, there was a significant trend for the occurrence of the other three interactive states to augment across sessions.

\subsection{Child Engagement and Child-System-Adult Interactivity}

Considering each interactive state as a separate unit of analysis, we could verify that, when there was no ongoing social interaction between the child and the adult, instances of the 'nonengaged' category were clearly predominant (RFs median $=.888)$. Within this interactive state few instances of 'active engagement' $($ RFs median $=.066)$ and of 'sparsely active engagement' $($ RFs median $=.035)$ levels could be observed. The Wilcoxon matched pairs signed ranks test showed that differences between the first (nonengaged) and the other two distributions (active and sparsely active) were significant $(Z=-2.20 ; \mathrm{p}<.03)$.

Only two levels of engagement occurred during the 'adult as model' interactive state: the 'attentional engagement' (RFs median $=.682$ ) and the 'nonengaged' (RFs median $=.318$ ). Compared to the second, the first level has appeared with higher incidence in all six participants. Such predominance reached statistical significance, as indicated by a two-tailed sign test $(\mathrm{p}<.04)$. 
Concerning 'Child-Adult as peers in the system', there was a clear prevalence ( $\mathrm{p}<.04$; two-tailed sign test) of the 'active engagement' level (RFs median =.902) as compared to the 'sparsely active engagement' one (RFs median = .099). During this state no instances of the 'nonengaged' and of the 'attentional engagement' levels were registered. While 'Child as performer/Adult as public' was an ongoing state, 'active engagement' (RFs median $=.872$ ) appeared more frequently than the 'sparsely engagement' (RFs median = .149) level did. Because the opposite trend was observed in one of the six participants (RF for active engagement $=.423$; RF for sparsely active engagement $=.577$ ), the difference could not reach statistical significance ( $p>$.217; two-tailed sign test). Since we defined engagement as the amount of time children spend interacting with the system, no engagement behaviour was coded during the so-called state 'ChildAdult as peers outside the system' state.

\subsection{Quality of Movement and Child-System-Adult Interactivity}

Incidence of spontaneous gestures (RFs median $=.897)$ was higher than incidences of imitative gestures (RFs median $=.071)$ and of creative movements (RFs median $=.033)$. In order to explore possible relations between the quality of movement and CSA interactivity, we have considered the total amount of time intervals assigned to the category of 'creative movement'. Proportional occurrences were, then, calculated taking into account the following interactive states: (A) 'no social interaction', (B) 'adult as model', (C) 'child as performer/adult as public', and (D) 'child-adult as peers in the system' (as CS interactivity could not be observed in 'child-adult as peers outside the system' we ignored this state). The same procedure was followed for the categories of 'imitative gesture' and 'spontaneous gesture'.

Instances of 'creative movement' appeared mostly while the child and the adult were playing 'as peers in the system' (see Fig. 1). Compared to each of the other interactive states this incidence was significantly higher in all cases $(\mathrm{p}<.04$; two-tailed sign test). Creative movements were also more frequent when the child was acting in front of the 'adult as public' than during the states of 'adult as model' and of 'no social interaction' (in both cases: $\mathrm{p}<.04$; two-tailed sign test).

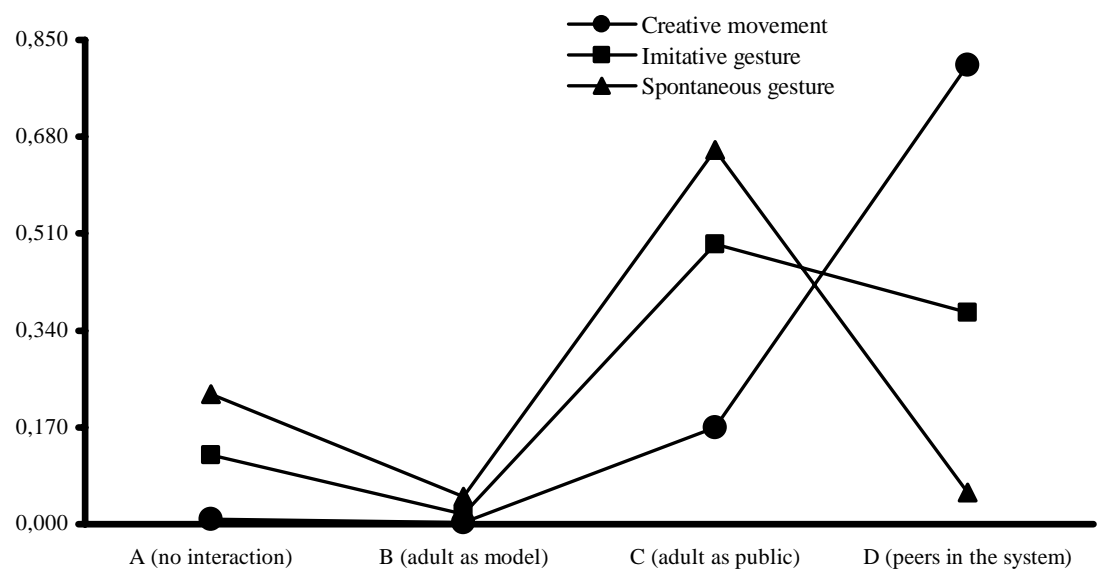

Figure 1. Medians (RFs) for creative movements, imitative gestures, and spontaneous gestures as function of the considered interactive states.

Imitative gestures were more frequent within 'child as performer/adult as public' and 'child-adult as peers in the system' than within time segments when 'no social interaction' or 'adult as model' were observed $((\mathrm{p}<$ .04; two-tailed sign test). Differences concerning spontaneous gestures between the first, the third and the fourth interactive state were not statistically significant.

\subsection{Affect Expressions and Child-System-Adult Interactivity}

Time intervals assigned to each one of the interactive states were the measures (units of analysis) from which RFs for 'positive affect', 'negative affect', and 'avoidance' have been extracted. Avoidant behaviour was rarely observed. Only three children expressed few instances of avoidance while the adult was acting as a model.

Expressions of negative affect were also seldom observed and merely occurred during the following two interactive states: 'no social interaction' and 'adult as model'. 
Concerning the occurrence of positive affect, instances appeared in the states of 'adult as model' (RFs median $=.290$ ), 'child as performer/adult as public' (RFs median $=.148$ ), 'child-adult as peers in the system' $($ RFs median $=.464)$, and of 'child-adult as peers outside the system' $($ RFs median $=.571)$.

The Friedman two-way analysis of variance test indicated that differences were overall statistically significant $(\mathrm{p}<.006)$. Pair wise comparisons by the Wilcoxon test revealed that incidences of positive affect differed between the states of 'child-adult as peers in the system' and of 'adult as model' $(Z=-1.99 ; p<.05)$ as well as between 'child-adult as peers in the system' and 'child as performer/adult as public' ( $Z=-2.20 ; p<$ .03). These last two distributions (adult as model and child as performer) differed also significantly from the distribution within the state of 'child-adult as peers outside the system' (respectively: $\mathrm{Z}=1.99 ; \mathrm{p}<.05$ and $\mathrm{Z}=$ $-2.20 ; \mathrm{p}<.03$ ). Differences between events assigned to the states of child-adult as peers in the system and outside the system did not reach statistic significance $(\mathrm{p}>.11)$.

\subsection{Communication and Child-System-Adult Interactivity}

Considering the total amount of time intervals assigned to the categories of 'non-verbal communication' and of 'verbal communication' as the unit of analysis, we explored relations between communicative behaviour and CSA interactivity. One of the participants revealed instances of verbal and non-verbal communication in most of the interactive states. The other participants only demonstrated these behaviours during the states of child-adult as peers (in or outside the system). Both verbal and non-verbal communication behaviours were more frequent in the interactive state of 'adult-child as peers outside the system' than in the other referred state (Fig. 2).

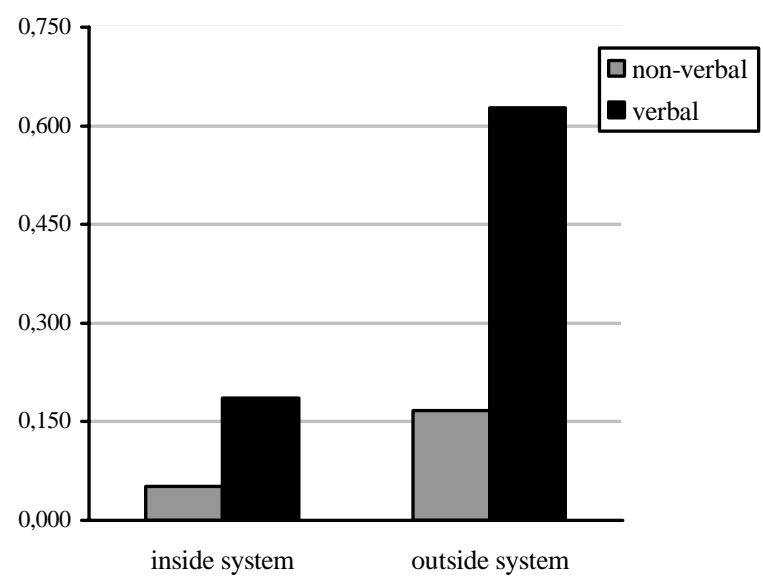

Figure 2. Medians (RFs) for non-verbal and verbal communication behaviours as function of the considered interactive states.

Concerning non-verbal and verbal communication, differences between occurrences in both interactive states were significant $(\mathrm{p}<.04$; two-tailed sign test $)$.

\section{DISCUSSION}

The research base for providing (re)habilitation services to children with disabilities has evolved dramatically in the past few years. There is now more complete and detailed information about how humans learn and develop. Contemporary views stress that development occurs across multiple interconnected behavioural levels (Lopes-dos-Santos, 2000; Overton, 2003), and several integrative approaches describe how the social relationships of children with adults and peers provide the critical foundations for learning and development (Spiker, Hebbeler, et al., 2005).

The recognition that social contexts exert a strong influence on child behaviour led us to explore interpersonal dynamics between children with severe neuromotor disabilities and the adult monitoring their performance in a multi-sensorial Aesthetic Resonance Environment. Results showed that adult-child interactions provided an organizing structure for children activities.

Child engagement and expressions of positive affect increased while children and the adult were participating in shared activities, namely when they were acting 'as peers inside the system'. This interactive state seemed to make visual modelling more efficient, affording the necessary scaffolding for the child to 
'reinvent' own gestures and to transform them into creative movements. A specific form of modelling - a scenario in which the adult suggested trajectories by moving his/her hand over the screen - appeared as a particularly powerful technique to catalyse subsequent new gestures. Both imitative and creative movements were evoked when the child seemed to act 'as a performer in front of the adult'. Furthermore, instances of verbal communication were mainly observed when the child and the adult related with each other 'as peers outside the system'. On the other side, if the adult was demonstrating the system, children were likely to remain quiet and 'attentively engaged'.

Analysis revealed that joint-activities increased from the first to the fourth observation. There was indeed a trend for instances of 'child/adult as peers' (inside or outside the system) to emerge more frequently across sessions. Even though the adult used some facilitation strategies to initiate and maintain the occurrence of such interaccional states, the portrayed results are likely to reflect the effects of practice and learning. Children acquired a progressive capacity to handle the system. Since social behaviour is fuelled by feelings of efficacy following self-recognized achievement of mastery (Shonkoff \& Philips, 2000), the enhanced sense of control may have encouraged them to participate in child-adult shared activities.

Specific and new intervention perspectives are revealed when the interpersonal dimension within Aesthetically Resonant Environments is explored. Recognizing the importance of such dimension underscores the potential advantages of integrating social mediation procedures in (re)habilitation frameworks inspired on the concept of 'aesthetic resonance'.

Disabilities may lead to general developmental problems that become increasingly great, as individuals grow older. Regarding neuromotor-disabled children, evidence shows that most of them do not easily engage in social interactions with others (e.g., Lopes-dos-Santos \& Fuertes, 2005). Subsequent delays in the social and communicative domains are, in turn, likely to reduce children's ability to learn from social environments (Wolery, 2000). The detrimental impact of such disability-amplifying processes can be minimized if interpersonal phenomena that seem to be unique to CSA interactivity are assessed and targeted as intervention goals. Likewise, the same processes that frame social behaviour as a relevant factor in neuromotor (re)habilitation should be seen as possible tools for intervention in other kinds of disabilities namely in those where social behaviour is itself the primary target (e.g., autistic spectrum disorders).

Practices of embedding interpersonal dynamics in CS interactivity call for more research and further refinements. We are currently conducting an investigation on facilitation strategies to enhance adult-child interactions. Conceptually guided by principles from the 'Zone of Proximal Development' construct (Vygotsky, 1978), this work aims at the elaboration of guidelines to improve quality of CSA interactivity within Aesthetically Resonant Environments.

Acknowledgements: Thanks to Carlos Araújo for his expertise in preparing digital versions of the videos. Thanks to the participants and their parents for their unconditional willingness to collaborate in this study. Thanks to the Rehabilitation Center 'Rui Abrunhosa' and their staff for the provided collaboration. This work was financially supported by a grant from Secretariado Nacional para a Reabilitação e Integração das Pessoas com Deficiência, Program CITE 2004.

\section{REFERENCES}

A. L. Brooks, (2004a), Soundscapes, In Inhabited Information Spaces: Living With Your Data (D. N. Snowdon, E. F. Churchill \& E. Frécon, Eds), Springer-Verlag: London, pp.89-99.

A. L. Brooks, (2004b), Soundscapes: Multi-sensory reciprocity through subliminal (non) control, Proc. 6th Int. Research Conference: Consciousness Reframed: Qi and Complexity, Beijing, pp. 37-44.

A. L. Brooks, A. Camurri, N. Canagarajah. and S. Hasselblad (2002), Interaction with shapes and sounds as a therapy for special needs and rehabilitation, Proc. $4^{\text {th }}$ Int. Conference on Disability, Virtual Reality and Associated Technologies, Veszprém, pp. 205-212.

A. L. Brooks and S. Hasselblad, S., (2004), Creating aesthetically resonant environments for the handicapped, elderly and rehabilitation: Sweden, Proc. $5^{\text {th }}$ Int. Conference on Disability, Virtual Reality and Associated Technologies, Oxford, pp. 191-198.

R. Kizony, N. Katz and P. L. Weiss (2004), Virtual reality based intervention in rehabilitation: Relationship between motor and cognitive abilities and performance within virtual environments for patients with stroke, Proc. $5^{\text {th }}$ Int. Conference on Disability, Virtual Reality and Associated Technologies, Oxford, pp. 19-26.

Proc. $6^{\text {th }}$ Int Conf. Disability, Virtual Reality \& Assoc. Tech., Esbjerg, Denmark, 2006

(C) 2006 ICDVRAT/University of Reading, UK; ISBN 0704998653 
P. Lopes-dos-Santos (2000), The organizational perspective in human development research: A metanarrative framework for guiding transdisciplinary practices in early childhood intervention, Proc. Int. Conference: The Social and Cultural Worlds of the Child, Braga, pp. 130-138.

P. Lopes-dos-Santos and M. Fuertes (2005), Developmental risk and attachment, In Development: Family and Educational Contexts (J. Bairrão, Ed.), Livpsic, Porto, pp.172-199.

W. Overton (2003), Development across the lifespan, In Handbook of Psychology: Vol 6. Developmental Psychology (R. Lerner, M. Easterbrooks, J. Mistry, Vol. Eds.), Wiley, New Jersey, pp. 13-42.

E. Petersson and A. L. Brooks (2006), Virtual and physical toys: Open-ended features for non-formal learning, CyberPsychol. and Behav., 9, pp. 196-199.

B. Rogoff (1993), Children's guided participation and participatory appropriation in social activity. In, Development in Context: Acting and Thinking in Specific Environments (R Wozniak \& K. Fisher, Eds.), Erlbaum, New Jersey, pp. 121-143.

B. Rogoff (2003), The Cultural Nature of Human Development, Oxford University Press, New York.

J. P. Shonkoff and D. A. Phillips (2000), From Neurons to Neighborhoods: The Science of Early Childhood Development, National Academy Press, Washington.

D. Spiker, K. Hebbeler and S. Mallik (2005), Developing and implementing early intervention programs for children with established disabilities, In The Developmental Systems Approach to Early Intervention (M. Guralnick, Ed.), Paul Brookes, Baltimore, pp. 305-349.

L. S. Vygotsky (1978), Mind in Society: The Development of Higher Psychological Processes, Harvard University Press, Cambridge, MA.

J. V. Wertsch (1985), Culture, Communication, and Cognition: Vygotskian Perspectives, Cambridge University Press, New York.

M. Wolery (2000), Behavioral and educational approaches to early intervention, In Handbook of Early Childhood Intervention (J. P. Shonkoff \& S. M. Meisels, Eds.), Cambridge University Press, New York, pp. 179-203. 